OCU-PHYS-245

April 2006

July 2006 (Revised)

\title{
Renormalization in Coulomb-gauge QCD within the Lagrangian formalism
}

\author{
A. NiÉGAWA \\ Graduate School of Science, Osaka City University \\ Sumiyoshi-ku, Osaka 558-8585, Japan
}

\begin{abstract}
We study renormalization of Coulomb-gauge QCD within the Lagrangian, secondorder, formalism. We derive a Ward identity and the Zinn-Justin equation, and, with the help of the latter, we give a proof of algebraic renormalizability of the theory. Through diagrammatic analysis, we show that, in the strict Coulomb gauge, $g^{2} D^{00}$ is invariant under renormalization. ( $D^{00}$ is the time-time component of the gluon propagator.)
\end{abstract}




\section{Introduction}

In nonabelian gauge theories, among a variety of gauge choices, the Coulomb gauge is one of the most important ones. The theories with this gauge are described in terms of physical fields, so that the unitarity is manifest. Within the Hamiltonian, first-order, formalism, formal or algebraic renormalizability of the Coulomb gauge has been studied in [1]. Since then, introducing an interpolating gauge, which interpolate between a covariant gauge and the Coulomb gauge, Baulieu and Zwanziger have proved algebraic renormalizability of the theory [2]. In taking the Coulombgauge limit, a phase-spaces representation is used there. Despite its importance, the proof of renormalizability of the Coulomb gauge within the Lagrangian, second-order, formalism per se is still lacking, to which the present paper is devoted.

In this paper, we are concerned about Coulomb gauge QCD, whose Lagrangian density is obtained by adding the gauge fixing term, $\mathcal{L}_{\text {G.F. }}=-\left(\partial_{i} A_{a}^{i}\right)\left(\partial_{j} A_{a}^{j}\right) / 0^{+}$, to the QCD Lagrangian density. In the Coulomb gauge, there is an inherent problem of appearance of 'energy divergences', which are characteristic of an instantaneous closed ghost- and $A_{0}$-loops:

$$
\int \frac{d p_{0}}{2 \pi} F(\mathbf{p}, \ldots)
$$

where $F$ is independent of $p_{0}$, the temporal component of the four-vector $P^{\mu}=\left(p_{0}, \mathbf{p}\right)$ and "..." indicates a set of external momenta. There also appear ill-defined integrals of the forms,

$$
\begin{aligned}
& \int \frac{d p_{0}}{2 \pi} \frac{p_{0}}{p_{0}^{2}-p^{2}+i 0^{+}} G(\mathbf{p}, \ldots) \\
& \int \frac{d p_{0}}{2 \pi} \frac{d q_{0}}{2 \pi} \frac{p_{0}}{p_{0}^{2}-p^{2}+i 0^{+}} \frac{q_{0}}{q_{0}^{2}-q^{2}+i 0^{+}} H(\mathbf{p}, \mathbf{q}, \ldots),
\end{aligned}
$$

where $G(H)$ is independent of $p_{0}\left(p_{0}\right.$ and $\left.q_{0}\right)$.

A numerous work has been devoted to the energy divergence problem and, by now, the following results are established.

1) In the Hamiltonian, phase-space, first order, formalism, energy divergences like (1) do not appear [3] in the first place.

2) Using the correspondence formula which equates amplitudes in a covariant gauge to those in a gauge without ghosts, Cheng and Tsai [4] "indirectly" showed 
that, when all relevant contributions are added, cancellation occurs between the energy divergences $(1)$.

3) With the help of an interpolating gauge, which interpolates between a covariant gauge and the Coulomb gauge, in the phase-space formalism, it has been shown by Baulieu and Zwanziger [2] that the cancellation occurs between different contributions which turn out to energy-divergent ones in the Coulomb-gauge limit (see, also, [3]).

4) Ill-defined integrals like (2) can be set equal to zero [5] and another type of illdefined integrals, Eq. (3), are connected $[3,5]$ with the so-called $V_{1}+V_{2}$ terms of Christ and Lee [6], which arise through correct treatment of operator ordering in the Hamiltonian.

5) It has been shown in [7] that the cancellation of energy divergences and renormalizability is compatible in an example in which quark-loop subgraphs are inserted into the second-order gluon self-energy graphs: As mentioned in 1) above, in the phase-space formalism, two integrals over the internal energies converge. However, in relation to renormalization, energy-divergences re-appear. Thanks to the Ward identity, these energy-divergent contributions cancel out.

Therefore, when perturbative computations in the present Lagrangian formalism are properly handled, cancellation should occur between the energy divergences. We are not concerned, in this paper, with the energy-divergence issue anymore.

As stated above, we are are interested in the Coulomb gauge QCD. Nevertheless, we proceed, as far as possible, with more general gauge choice, $\mathcal{L}_{\text {G.F. }}=-\left(\partial_{i} A_{a}^{i}\right)^{2} /(2 \alpha)$ with arbitrary $\alpha$, which is usually called the Coulomb gauge. The "genuine" Coulomb gauge, which is obtained by taking the limit $\alpha \rightarrow 0^{+}$, is called the strict Coulomb gauge. Incidentally, in the case of $\alpha \neq 0$ Coulomb gauge, when compared to the strict Coulomb gauge, much worse energy divergences arise (cf. Eq. (5) below), so that, at the present stage, such a gauge is useless for practical perturbative calculations.

In $\S 2$, we present a Ward identity and the Zinn-Justin equation, which are derived in Appendix A. In $\S 3$, on the basis of the Zinn-Justin equation, we construct renormalization counterterms in a recursive way. In $\S 4$, we prove an algebraic renormalizability of the theory and obtain the identities between the renormalization constants. With 
the aid of the Ward identity, we obtain an additional identity $\tilde{Z}_{1}=Z_{12} / Z_{31}$ (Eq. (41)). We then show that, in the strict Coulomb gauge, $g^{2} D^{00}$ ( $D^{00}$ is the time-time component of the gluon propagator) is invariant under renormalization. In $[1,2]$, this proposition is proved using the Ward identity. In contrast, in the present Lagrangian formalism, use of the Zinn-Justin equation is sufficient for proving this. For illustrative purpose, the forms of some one-loop renormalization constants in the strict Coulomb gauge are also displayed. $\S 5$ is devoted to summary. Appendix A briefly describes a derivation of the Ward identity and the Zinn-Justin equation. In Appendix $\mathrm{B}$, using the identity that is dereived from the Zinn-Justin equation, we obtain, in the strict Coulomb gauge, an additional identities $\tilde{Z}_{1}=D^{\prime}=1$, Eq. (B.5). In Appendix $\mathrm{C}$, we briefly describe a derivation of some one-loop renormalization constants in the strict Coulomb gauge. Some formal diagrammatic analyses are summarized in Appendix D. In particular, we show that, in the strict Coulomb gauge, $\bar{\eta} A \eta$-vertex (cf. Eq. (4)) is not a renormalization part, so that $\tilde{Z}_{1}=1$ in the minimal subtraction scheme in dimensional regularization.

\section{Ward identity and Zinn-Justin equation}

As the content of this section is standard, we describe briefly. Greek indices $\mu, \nu, \ldots$ run over $0,1,2,3$, while Latin indices $i, j$ run over $1,2,3$. We use $P^{\mu}$ for denoting a four vector $P^{\mu}=\left(p^{0}, \mathbf{p}\right)$ and $p^{j}$ for denoting a three vector.

The effective Lagrangian density of Coulomb-gauge QCD with one quark flavor (generalization to the case of several quarks is straightforward) reads

$$
\begin{aligned}
& \mathcal{L}_{\text {eff }}=\tilde{\mathcal{L}}_{\text {eff }}-\frac{1}{2 \alpha} \mathcal{F}_{a} \mathcal{F}_{a} \quad\left(\mathcal{F}_{a}[A ; x]=\partial_{i} A_{a}^{i}(x)\right), \\
& \tilde{\mathcal{L}}_{\text {eff }}=-\frac{1}{4} F_{a}^{\mu \nu} F_{a \mu \nu}+\bar{\psi}\left(i \not D-m-g t_{a} A_{a}\right) \psi+\partial_{i} \bar{\eta}_{a} D_{a b}^{i}(x) \eta_{b},
\end{aligned}
$$

where $F_{a}^{\mu \nu}=\partial^{\mu} A_{a}^{\nu}-\partial^{\nu} A_{a}^{\mu}-g f_{a b c} A_{b}^{\mu} A_{c}^{\nu}, t_{a}=\lambda_{a} / 2$, and $D_{a b}^{\mu}(x)=\delta_{a b} \partial^{\mu}+g f_{a b c} A_{c}^{\mu}(x)$. Generalization to other nonabelian gauge theories is straightforward. In the Lagrangian formalism adopted here, the fields propagators can be extracted from the bilinear (with respect to the fields) terms of $\mathcal{L}_{\text {eff }}$ in Eq. (4). For the purpose of later use, among the propagators, we only display the forms of the gluon propagator $\Delta^{\mu \nu}(Q)$ (see, also, $\left.[8,9]\right)$ and FP-ghost propagator $\tilde{\Delta}(Q)$, together with $\bar{\eta}_{a}(Q) A_{b}^{i} \eta_{c^{-}}$ 
vertex factor $\mathcal{V}_{a b c}^{i}(Q)$ :

$$
\begin{aligned}
\Delta^{\mu \nu}(Q) & =g_{i}^{\mu} g_{j}^{\nu} \frac{\delta^{i j}-q^{i} q^{j} / q^{2}}{Q^{2}+i 0^{+}}+\frac{n^{\mu} n^{\nu}}{q^{2}}-\alpha \frac{Q^{\mu} Q^{\nu}}{q^{4}}, \\
\tilde{\Delta}(Q) & =-\frac{1}{q^{2}} \\
\mathcal{V}_{a b c}^{i}(Q) & =g f_{a b c} q^{i} .
\end{aligned}
$$

Propagators are diagonal in color space, so that the color indices are suppressed.

The gauge-field part and the quark part of $\tilde{\mathcal{L}}_{\text {eff }}$ is invariant under the infinitesimal gauge transformation: $A_{a}^{\mu} \rightarrow A_{a}^{\mu}+D_{a b}^{\mu}(x) \epsilon_{b}(x), \psi \rightarrow \psi-i g \epsilon_{a} t_{a} \psi$, and $\bar{\psi} \rightarrow \bar{\psi}+i g \bar{\psi} \epsilon_{a} t_{a}$. $\mathcal{L}_{\text {eff }}$ is invariant under the BRST transformation of the fields

$$
\begin{aligned}
\delta A_{a}^{\mu}(x) & =D_{a b}^{\mu}(x) \eta_{b} \zeta \equiv s A_{a}^{\mu} \zeta \\
\delta \eta_{a}(x) & =-\frac{g}{2} f_{a b c} \eta_{b}(x) \eta_{c}(x) \zeta \equiv s \eta_{a} \zeta, \\
\delta \psi & =i g \eta_{a} t_{a} \psi \zeta \equiv s \psi \zeta, \quad \delta \bar{\psi}=i g \bar{\psi} \eta_{a} t_{a} \zeta \equiv s \bar{\psi} \zeta \\
\delta \bar{\eta}_{a}(x) & =\frac{1}{\alpha} \mathcal{F}_{a}[A ; x] \zeta \equiv s \bar{\eta}_{a} \zeta .
\end{aligned}
$$

Here $\zeta$ is an $x$-independent infinitesimal Grassmann number with the same ghost number as $\bar{\eta}_{a}$. $\mathcal{L}_{\text {eff }}$ is not invariant under the Lorentz transformation but is invariant under the spatial rotation. Then, we treat the spatial component $A_{a}^{i}$ and the temporal component $A_{a}^{0}$ of $A_{a}^{\mu}$ separately. We let $\chi^{n}$ run over the fields, $A_{a}^{i}, A_{a}^{0}, \eta_{a}, \psi$, and $\bar{\psi}$, and introduce a set of c-number external sources $K_{n}(x)$ that couple to $s \chi^{n}[\chi ; x]$, $K_{n}=\left(K_{a}^{i}, K_{a}^{0}, K_{\eta_{a}}, K_{\psi}, K_{\bar{\psi}}\right)$.

Quantum effective action $\Gamma$ is defined by the following implicit functional integrodifferential equation:

$$
\begin{aligned}
e^{i \Gamma[\chi, \bar{\eta}, K]}= & \int \mathcal{D}\left(\chi^{\prime}, \bar{\eta}^{\prime}\right) \exp \left[i \int d ^ { 4 } y \left(\mathcal{L}_{\mathrm{eff}}\left(\chi^{\prime}, \bar{\eta}^{\prime}\right)+K_{n} s \chi^{\prime n}\right.\right. \\
& \left.\left.-\frac{\delta_{R} \Gamma}{\delta \chi^{n}}\left(\chi^{\prime n}-\chi^{n}\right)-\frac{\delta_{R} \Gamma}{\delta \bar{\eta}_{a}}\left(\bar{\eta}_{a}^{\prime}-\bar{\eta}_{a}\right)\right)\right]
\end{aligned}
$$

where ' $R$ ' denotes right differentiation. We show in Appendix A that

$$
\Gamma[\chi, \bar{\eta}, K] \text { depends on } \bar{\eta} \text { only through } K_{a}^{j}+\partial^{j} \bar{\eta}_{a} .
$$

We introduce $\tilde{\Gamma}$ through

$$
\tilde{\Gamma}=\Gamma+\frac{1}{2 \alpha} \int d^{4} y \mathcal{F}^{2}[A ; y]
$$


The Ward identity, which is derived in Appendix A, reads

$$
\int d^{3} x\left[\frac{\delta_{R} \tilde{\Gamma}}{\delta \chi^{n}} \frac{\delta_{L} \tilde{\Gamma}}{\delta K_{n}}\right](x)=\partial_{0} \int d^{3} x\left[\eta_{a} \frac{\delta_{R} \tilde{\Gamma}}{\delta A_{a}^{0}}+K_{a}^{0} \frac{\delta_{L} \tilde{\Gamma}}{\delta K_{\eta_{a}}}\right](x),
$$

where ' $L$ ' denotes left differentiation. Integration over $x_{0}$ yields the Zinn-Justin equation,

$$
0=\int d^{4} x\left[\frac{\delta_{R} \tilde{\Gamma}}{\delta \chi^{n}} \frac{\delta_{L} \tilde{\Gamma}}{\delta K_{n}}\right](x) \equiv(\tilde{\Gamma}, \tilde{\Gamma}) .
$$

The leading term (in the loop expansion) of $\tilde{\Gamma}$ is

$$
\tilde{\Gamma}_{0}[\chi, \bar{\eta}, K]=\int d^{4} x\left(\tilde{\mathcal{L}}_{\text {eff }}[\chi, \bar{\eta}, ; x]+K_{n} s \chi^{n}[\chi ; x]\right),
$$

which is invariant under the transformation (8) - (10). It should be noted that the fields $\chi^{n}$ and $\bar{\eta}$ in $\tilde{\Gamma}_{0}$ are the renormalized ones.

\section{Recursive construction of counterterms}

In this section, we construct the renormalization counterterms that preserve the symmetry condition (16). We follow the procedure in standard text books [10, 11], so that we briefly describe.

Let us use dimensional regularization by continuing spacetime dimensions from 4 to $d$. We employ the loop expansion for $\tilde{\Gamma}, \tilde{\Gamma}=\sum_{N=0}^{\infty} \tilde{\Gamma}_{N}$ and decompose $\tilde{\Gamma}_{N}$ as $\tilde{\Gamma}_{N}=\tilde{\Gamma}_{R N}+\tilde{\Gamma}_{N}^{(\infty)}$, where $\tilde{\Gamma}_{N}^{(\infty)}$ is the ultra-violet (UV) divergent contribution, i.e., it diverges in the limit $d \rightarrow 4$. We adopt the minimal subtraction scheme.

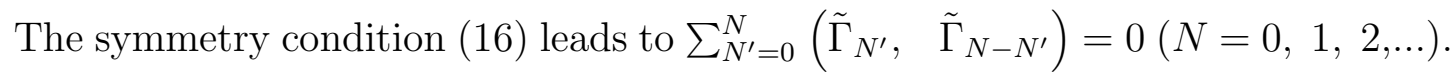
We proceed in a recursive way. We assume that, for all $M \leq N-1$, all UV-divergent contributions from $M$-loop diagrams have been cancelled by counterterms $\tilde{\Gamma}_{M}^{(\infty)}(M$ $=1,2, \ldots, N-1$ ). (This recursive procedure is justified a posteriori in $\S 4$.) Then UV infinities can appear only in the $N^{\prime}=0$ and $N^{\prime}=N$ terms, and the infinite part of

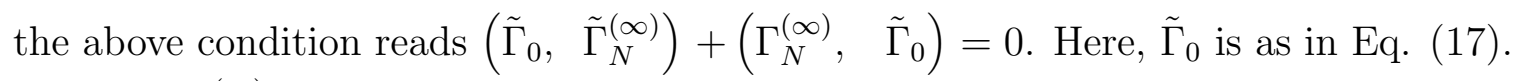
We write $\tilde{\Gamma}_{N}^{(\infty)}$ as

$$
\tilde{\Gamma}_{N}^{(\infty)}[\chi, \bar{\eta}, K]=\int d^{4} x\left(\tilde{l}_{N}[\chi ; x]+K_{n} \mathcal{D}_{N}^{n}[\chi ; x]\right)
$$


where $\tilde{l}_{N}$ and $\mathcal{D}_{N}^{n}$ are local functions of $\chi^{n}$ and $\bar{\eta}$ and their derivatives [11]. For the time being, we drop the suffix $N$. Substitution of Eqs. (17) and (18) gives

$$
\begin{aligned}
& \int d^{4} x\left(\frac{\partial_{R} \tilde{\mathcal{L}}_{\text {eff }}[\chi, \bar{\eta} ; x]}{\partial \chi^{n}(x)} \mathcal{D}^{n}[\chi ; x]+\frac{\partial_{R} \tilde{l}[\chi, \bar{\eta} ; x]}{\partial \chi^{n}(x)} s \chi^{n}[\chi ; x]\right)=0 \\
& \int d^{4} x\left(\frac{\partial_{R} s \chi^{m}[\chi ; x]}{\partial \chi^{n}(x)} \mathcal{D}^{n}[\chi ; x]+\frac{\partial_{R} \mathcal{D}^{m}[\chi ; x]}{\partial \chi^{n}(x)} s \chi^{n}[\chi ; x]\right)=0
\end{aligned}
$$

From here on, we follow the procedure in Sec. 17 of [11]. We introduce

$$
\begin{aligned}
\tilde{\Gamma}^{(\epsilon)}[\chi, \bar{\eta}] & =\int d^{4} x\left(\tilde{\mathcal{L}}_{\mathrm{eff}}[\chi, \bar{\eta} ; x]+\epsilon \tilde{l}[\chi, \bar{\eta} ; x]\right) \\
& \equiv \int d^{4} x \tilde{\mathcal{L}}^{(\epsilon)}[\chi, \bar{\eta} ; x], \\
\Delta^{(\epsilon) n}[\chi ; x] & =s \chi^{n}[\chi ; x]+\epsilon \mathcal{D}^{n}[\chi ; x],
\end{aligned}
$$

with $\epsilon$ infinitesimal for technical reason. Then, Eqs. (19) says that $\tilde{\Gamma}^{(\epsilon)}$ is invariant under the transformation,

$$
\chi^{n}(x) \rightarrow \chi^{n}(x)+\Delta^{(\epsilon) n}[\chi ; x] \zeta
$$

while Eq. (20) tells us that this transformation is nilpotent.

The most general form of the transformation (23) is

$$
\begin{aligned}
A_{a}^{i} & \rightarrow A_{a}^{i}+\left[B_{a b}^{(\epsilon)} \partial^{i} \eta_{b}-g D_{a b c}^{(\epsilon)} A_{b}^{i} \eta_{c}\right] \zeta, \\
A_{a}^{0} & \rightarrow A_{a}^{0}+\left[B_{a b}^{(\epsilon)^{\prime}} \partial^{0} \eta_{b}-g D_{a b c}^{(\epsilon)^{\prime}} A_{b}^{0} \eta_{c}\right] \zeta, \\
\eta_{a} & \rightarrow \eta_{a}-\frac{g}{2} E_{a b c}^{(\epsilon)} \eta_{b} \eta_{c} \zeta \\
\psi & \rightarrow \psi+i g \eta_{a} T_{a}^{(\epsilon)} \psi \zeta
\end{aligned}
$$

where $B_{a b}^{(\epsilon)}, B_{a b}^{(\epsilon)^{\prime}}, D_{a b c}^{(\epsilon)}, D_{a b c}^{(\epsilon)^{\prime}}$, and $E_{a b c}^{(\epsilon)}\left(=-E_{a c b}^{(\epsilon)}\right)$ are constants, and $T_{a}^{(\epsilon)}$ is some matrix acting on the quark field. Here $D_{a b c}^{(\epsilon)}=\left(D_{a b c}^{(\epsilon)}\right)_{N}=f_{a b c}+\epsilon\left(D_{a b c}\right)_{N}, T_{a}^{(\epsilon)}=$ $\left(T_{a}^{(\epsilon)}\right)_{N}=t_{a}+\epsilon\left(T_{a}\right)_{N}$, etc. Even if we introduce different $T_{a}^{(\epsilon)}$ 's for different components of $\psi$, we will have the same result as the one obtained below. Imposing the condition of nilpotence, we obtain $E_{a b c}^{(\epsilon)}=D_{a b c}^{(\epsilon)}=D_{a b c}^{(\epsilon)^{\prime}}=C^{(\epsilon)} f_{a b c}, B_{a b}^{(\epsilon)}=(C D)^{(\epsilon)} \delta_{a b}$, $B_{a b}^{(\epsilon)^{\prime}}=\left(C D^{\prime}\right)^{(\epsilon)} \delta_{a b}$, and $T_{a}^{(\epsilon)}=C^{(\epsilon)} t_{a}$, where $C^{(\epsilon)},(C D)^{(\epsilon)}$, and $\left(C D^{\prime}\right)^{(\epsilon)}$ are some constants: $C^{(\epsilon)}=C_{N}^{(\epsilon)}=1+\epsilon C_{N},(C D)^{(\epsilon)}=(C D)_{N}^{(\epsilon)}=1+\epsilon \sum_{N^{\prime}=0}^{N} C_{N^{\prime}} D_{N-N^{\prime}}$ $\left(C_{0}=D_{0}=1\right)$, etc. $\left(C_{M}\right.$ and $D_{M}(M<N)$ have already been determined at 
lower-order stages.) Thus, we have, with obvious notation,

$$
\begin{aligned}
A_{a}^{i} & \rightarrow A_{a}^{i}+\left[C D\left(\partial^{i} \eta_{a}-\tilde{g} f_{a b c} A_{b}^{i} \eta_{c}\right) \zeta\right]^{(\epsilon)} \\
A_{a}^{0} & \rightarrow A_{a}^{0}+\left[C D\left\{\left(D^{\prime} / D\right) \partial^{0} \eta_{a}-\tilde{g} f_{a b c} A_{b}^{0} \eta_{c}\right\} \zeta\right]^{(\epsilon)} \\
\eta_{a} & \rightarrow \eta_{a}+\left[C D\left(-\frac{1}{2} \tilde{g} f_{a b c} \eta_{b} \eta_{c} \zeta\right)\right]^{(\epsilon)}, \\
\psi & \rightarrow \psi+\left[C D\left(i \tilde{g} \eta_{a} t_{a} \psi \zeta\right)\right]^{(\epsilon)}
\end{aligned}
$$

where $\tilde{g} \equiv g / D$. Introducing $\tilde{A}_{a}^{0} \equiv\left(D / D^{\prime}\right) A_{a}^{0}$, one can rewrite Eq. (25) as

$$
\tilde{A}_{a}^{0} \rightarrow \tilde{A}_{a}^{0}+\left[C D\left(\partial^{0} \eta_{a}-\tilde{g} f_{a b c} \tilde{A}_{b}^{0} \eta_{c}\right) \zeta\right]^{(\epsilon)}
$$

$\tilde{\mathcal{L}}^{(\epsilon)}$ in Eq. (21) must be invariant under all the symmetries of the original Lagrangian, i.e., (1) invariance under the spatial rotation, (2) global gauge invariance, (3) antighost translation invariance, (4) ghost number conservation, and (5) quark number conservation. Furthermore, as stated in (13), $\tilde{\Gamma}$ does not involve $\partial_{0} \bar{\eta}$. (For completeness, this is proved in Appendix D through formal diagrammatic analyses.) Taking all of these facts, we find that the most general renormalizable interaction takes the form:

$$
\begin{aligned}
\tilde{\mathcal{L}}^{(\epsilon)} & =\tilde{\mathcal{L}}_{\mathrm{eff}}+\epsilon \tilde{l} \\
& =\mathcal{L}_{\psi A}^{(\epsilon)}+Z_{\eta}^{(\epsilon)}\left(\partial_{i} \bar{\eta}_{a}\right)\left(\partial^{i} \eta_{a}\right)-g d_{a b c}^{(\epsilon)}\left(\partial_{i} \bar{\eta}_{a}\right) A_{b}^{i} \eta_{c}
\end{aligned}
$$

where $Z_{\eta}^{(\epsilon)}, d_{a b c}^{(\epsilon)}$ are unknown constants, and $\mathcal{L}_{\psi A}^{(\epsilon)}$ is the renormalizable term that involves only the quark and gauge fields.

Imposition of the invariance under the transformation (24) - (27) yields

$$
\begin{aligned}
\tilde{\mathcal{L}}^{(\epsilon)}= & {\left[-\frac{Z_{31}}{4} \tilde{F}_{a}^{i j} \tilde{F}_{i j}^{a}-\frac{Z_{31}^{\prime}}{2} \tilde{F}_{a}^{0 i} \tilde{F}_{a}^{0 i}+Z_{21} \bar{\psi}\left(i \partial^{j}-\tilde{g} t_{a} A_{a}^{j}\right) \gamma_{j} \psi\right.} \\
& \left.+Z_{22} \bar{\psi}\left(i \partial^{0}-\tilde{g} t_{a} \tilde{A}_{a}^{0}\right) \gamma_{0} \psi-Z_{m}^{\prime} m \bar{\psi} \psi+\tilde{Z}_{3}\left(\partial_{i} \bar{\eta}_{a}\right)\left\{\partial^{i} \eta_{a}-\tilde{g} f_{a b c} A_{b}^{i} \eta_{c}\right\}\right]^{(\epsilon)} .
\end{aligned}
$$

The tilde on $\tilde{F}_{a}^{i j}$ indicates that the field strength is to be calculated using $\tilde{g}$ for $g$, while the tilde on $\tilde{F}_{a}^{0 i}$ indicates that the field strength is to be calculated using $\tilde{g}$ for $g$ and $\tilde{A}_{a}^{0}$ for $A_{a}^{0}$. All the constants appearing above are of the form, $Z_{31}^{(\epsilon)}=\left(Z_{31}^{(\epsilon)}\right)_{N}=$ $1+\epsilon\left(Z_{31}\right)_{N},\left(Z_{31} / D\right)^{(\epsilon)}=\left(Z_{31} / D\right)_{N}^{(\epsilon)}=1+\epsilon \sum_{N^{\prime}=0}^{N}\left(Z_{31}\right)_{N^{\prime}} D_{N-N^{\prime}}^{-1}\left(D_{0}^{-1}=1\right)$, etc. 
For obtaining further informations on the constants appearing in Eq. (29), we make following observations:

(i) Gauge-fixing independence of the locations of the physical poles (zeros) in the transverse-gluon- and the quark-propagators (two-point effective actions).

Kobes, Kunstattar, and Rebhan [12] showed this proposition to hold by using a set of identities that determine the gauge dependence of the effective action.

(ii) Argument in [12] goes as it is even in $d$-dimensional spacetime.

(iii) In the case of covariant gauge, the poles of the transverse-gluon propagator are at $p_{0}= \pm p\left(P^{2}=0\right)$ and the poles of the quark propagator are at $p_{0}=$ $\pm\left(p^{2}+m_{\mathrm{ph}}^{2}\right)^{1 / 2}\left(P^{2}=m_{\mathrm{ph}}^{2}\right)$, where $m_{\mathrm{ph}}$ is the physical quark mass. Thus, the dispersion relation of the transverse-gluon (quark) mode is Lorentz invariant, $P^{2}=0\left(P^{2}-m_{\mathrm{ph}}^{2}=0\right)$.

Our $Z^{(\epsilon)}$ 's are the ones that are computed in the minimal subtraction scheme in dimensional regularization. For taking the facts (i) - (iii) into account, the on-shell renormalization scheme (RS) should be employed. Let $\left(Z_{31}^{(\epsilon)}\right)_{\text {on }}$, etc. be the on-shell RS counterpart of $Z_{31}^{(\epsilon)}$, etc. Then, (i) - (iii) and the on-shell RS counterparts of Eq. (29) tell us that $\left(Z_{31}^{(\epsilon)}\right)_{\text {on }}=\left(Z_{31}^{(\epsilon)^{\prime}}\right)_{\text {on }}$ and $\left(Z_{21}^{(\epsilon)}\right)_{\text {on }}=\left(Z_{22}^{(\epsilon)}\right)_{\text {on }}$. Since $\left(Z_{31}^{(\epsilon)}\right)_{\text {on }}-Z_{31}^{(\epsilon)}$, etc. are UV finite, we obtain ${ }^{1}$

$$
Z_{31}^{(\epsilon)}=Z_{31}^{(\epsilon)^{\prime}}, \quad Z_{21}^{(\epsilon)}=Z_{22}^{(\epsilon)}\left(\equiv Z_{2}^{(\epsilon)}\right)
$$

\section{Algebraic renormalizability and the form of $\Gamma^{(\infty)}$}

In this section, we show the algebraic renormalizability of the Coulomb-gauge QCD. On the basis of the recursive construction in the last section, we deduce the form of $\Gamma^{(\infty)}$. Reviving the suffix $N$, setting $\epsilon=1$, and summing over $N$, we obtain from Eqs. (28) and (29) with Eq. (30),

$$
\tilde{\ell} \equiv \tilde{\mathcal{L}}_{\text {eff }}+\sum_{N=1}^{\infty} \tilde{l}_{N}
$$

\footnotetext{
${ }^{1}$ For arriving at the strict Coulomb gauge $(\alpha \rightarrow 0)$, one might encounter a subtle limiting procedure. The proposition (i) has been proved within the interpolating gauge in [2].
} 


$$
\begin{aligned}
= & -\frac{Z_{31}}{2}\left(\partial_{\mu} A_{a j} \partial^{\mu} A_{a}^{j}-\partial_{i} A_{a}^{j} \partial_{j} A_{a}^{i}\right)+Z_{32} \partial_{0} A_{a}^{i} \partial_{i} A_{a}^{0}-\frac{Z_{33}}{2} \partial_{i} A_{a}^{0} \partial^{i} A_{a}^{0} \\
& +g Z_{11} f_{a b c}\left(\partial_{i} A_{j}^{a}\right) A_{b}^{i} A_{c}^{j}+g Z_{12} f_{a b c} \partial^{0} A_{a}^{i} A_{b}^{0} A_{c i}+g Z_{13} f_{a b c} \partial^{i} A_{a}^{0} A_{b i} A_{c}^{0} \\
& -\frac{g^{2}}{4} Z_{41} f_{a b c} f_{a d e} A_{b i} A_{c j} A_{d}^{i} A_{e}^{j}-\frac{g^{2}}{2} Z_{42} f_{a b c} f_{a d e} A_{b}^{0} A_{c i} A_{d}^{0} A_{e}^{i} \\
& +\tilde{Z}_{3}\left(\partial_{i} \bar{\eta}_{a}\right) \partial^{i} \eta_{a}-g \tilde{Z}_{1} f_{a b c}\left(\partial_{i} \bar{\eta}_{a}\right) A_{b}^{i} \eta_{c} \\
& +Z_{2} \bar{\psi}(i \not \partial-(m-\delta m)) \psi-g Z_{\psi 11} \bar{\psi} t_{a} A_{a}^{j} \gamma_{j} \psi-g Z_{\psi 12} \bar{\psi} t_{a} A_{a}^{0} \gamma^{0} \psi
\end{aligned}
$$

where, with obvious notation, $Z_{31}=1+\sum_{N=1}^{\infty}\left(Z_{31}\right)_{N}$, etc., and

$$
\begin{array}{llll}
Z_{32}=D Z_{31} / D^{\prime}, & Z_{33}=D^{2} Z_{31} / D^{\prime 2}, & Z_{11}=Z_{31} / D, & Z_{12}=Z_{31} / D^{\prime}, \\
Z_{13}=D Z_{31} / D^{\prime 2}, & Z_{41}=Z_{31} / D^{2}, & Z_{42}=Z_{31} / D^{\prime 2}, & \tilde{Z}_{1}=\tilde{Z}_{3} / D, \\
Z_{\psi 11}=Z_{2} / D, & Z_{\psi 12}=Z_{2} / D^{\prime}, & \delta m=\left(1-Z_{m}^{\prime} / Z_{2}\right) m .
\end{array}
$$

Here, $D=1+\sum_{N=1}^{\infty} D_{N}$, etc. From these relations we obtain the identities:

$$
\begin{aligned}
& \frac{Z_{31}}{Z_{11}}=\frac{\tilde{Z}_{3}}{\tilde{Z}_{1}}=\frac{Z_{11}}{Z_{41}}=\frac{Z_{32}}{Z_{12}}=\frac{Z_{33}}{Z_{13}}=\frac{Z_{2}}{Z_{\psi 11}}(=D), \\
& \frac{Z_{31}}{Z_{12}}=\frac{Z_{12}}{Z_{42}}=\frac{Z_{32}}{Z_{13}}=\frac{Z_{2}}{Z_{\psi 12}}\left(=D^{\prime}\right) .
\end{aligned}
$$

Recalling Eq. (14), we finally have, for the density of $\left(\Gamma_{0}+\Gamma^{(\infty)}\right)_{K_{n}=0}$,

$$
\ell=\tilde{\ell}-\frac{Z_{31}}{2 \tilde{\alpha}}\left(\partial_{i} A_{a}^{i}\right)\left(\partial_{j} A_{a}^{j}\right)
$$

where $\tilde{\alpha}=Z_{31} \alpha$. We now define the bare fields and parameters according to

$$
\begin{array}{llll}
A_{B a}^{j}=Z_{31}^{1 / 2} A_{a}^{j}, & A_{B a}^{0}=Z_{33}^{1 / 2} A_{a}^{0}, & \eta_{B}=\tilde{Z}_{3}^{1 / 2} \eta, & \bar{\eta}_{B}=\tilde{Z}_{3}^{1 / 2} \bar{\eta} \\
\psi_{B}=Z_{2}^{1 / 2} \psi, & \bar{\psi}_{B}=Z_{2}^{1 / 2} \bar{\psi}, & g_{B}=Z_{11} Z_{31}^{-3 / 2} g, & \alpha_{B}=Z_{31} \alpha \\
m_{B}=m-\delta m & & &
\end{array}
$$

Then, from Eqs. (31) - (33) we see that $\ell$ may be regarded [10] as the initial Lagrangian density $\mathcal{L}_{\text {eff }}\left(A_{B}, \eta_{B}, \bar{\eta}_{B}, \psi_{B}, \bar{\psi}_{B} ; g_{B}, m_{B}, \alpha_{B}\right)$ written in terms of bare quantities.

By construction, the density $\tilde{\ell}$ in Eq. (31) is invariant under the transformation $\chi^{n} \rightarrow \chi^{n}+\Delta \chi^{n}$ that is obtained from Eqs. (24) - (27) by setting $\epsilon=1$ and summing over $N$, which reads, in obvious notation,

$$
\Delta A_{a}^{i}=S A_{a}^{i} \zeta=C\left(D \partial^{i} \eta_{a}-g f_{a b c} A_{b}^{i} \eta_{c}\right) \zeta=\frac{C}{\tilde{Z}_{1}} \tilde{Z}_{3}^{1 / 2}\left(\partial^{i} \eta_{B a}-g_{B} f_{a b c} A_{B b}^{i} \eta_{B c}\right) \zeta
$$




$$
\begin{aligned}
& \equiv Z_{a}^{i} s A_{B a}^{i} \zeta \\
\Delta A_{a}^{0} & =S A_{a}^{0} \zeta=C\left(D^{\prime} \partial_{0} \eta_{a}-g f_{a b c} A_{b}^{0} \eta_{c}\right) \zeta=\frac{C}{\tilde{Z}_{1}} \frac{Z_{11} \tilde{Z}_{3}^{1 / 2}}{Z_{12}}\left(\partial_{0} \eta_{B a}-g_{B} f_{a b c} A_{B b}^{0} \eta_{B c}\right) \zeta \\
& \equiv Z_{a}^{0} s A_{B a}^{0} \zeta \\
\Delta \eta_{a} & =S \eta_{a} \zeta=C\left(-\frac{1}{2} g f_{a b c} \eta_{b} \eta_{c}\right) \zeta=\frac{C}{\tilde{Z}_{1}} Z_{31}^{1 / 2}\left(-\frac{1}{2} g_{B} f_{a b c} \eta_{B b} \eta_{B c}\right) \zeta \equiv Z_{\eta_{a}} s \eta_{B a} \zeta \\
\Delta \psi & =S \psi \zeta=C\left(i g \eta_{a} t_{a} \psi\right) \zeta=\frac{C}{\tilde{Z}_{1}}\left(\frac{Z_{31} \tilde{Z}_{3}}{Z_{2}}\right)^{1 / 2}\left(i g \eta_{B a} t_{a} \psi_{B}\right) \zeta \equiv Z_{\psi} s \psi_{B} \zeta
\end{aligned}
$$

where use has been made of $\tilde{g}=g / D$ and Eq. (34). Then, from Eq. (18) with Eqs. (22), (23), and (35) we have

$$
\begin{aligned}
\tilde{\Gamma}_{0}+\sum_{N=1}^{\infty} \tilde{\Gamma}_{N}^{(\infty)} & =\int d^{4} x\left[\tilde{\ell}+K_{n} S \chi^{n}\right] \\
& \equiv \int d^{4} x\left(\tilde{\mathcal{L}}_{\mathrm{eff}}\left[\chi_{B}, \bar{\eta}_{B}\right]+K_{B n} s \chi_{B}^{n}\left[\chi_{B}^{n}\right]\right)
\end{aligned}
$$

where we have used $\tilde{\ell}=\tilde{\mathcal{L}}_{\text {eff }}\left[\chi_{B}, \bar{\eta}_{B}\right]$ (cf. after Eq. (34)). On the right-hand side of Eq. (36), $K_{a}^{i}$ and $\partial^{i} \bar{\eta}_{a}$ appear in the form,

$$
\tilde{Z}_{3}\left[C \tilde{Z}_{1}^{-1} K_{a}^{i}+\left(\partial^{i} \bar{\eta}_{a}\right)\right]\left[\partial_{i} \eta_{a}-\tilde{g} f_{a b c} A_{b i} \eta_{c}\right] .
$$

Recalling here the property (13), we obtain the relation $C=\tilde{Z}_{1}$. Thus, $\left(Z_{a}^{i}, Z_{a}^{0}, Z_{\eta_{a}}, Z_{\psi}, Z_{\bar{\psi}}\right)$ cannot be arbitrarily chosen but are determined uniquely:

$$
\begin{array}{ll}
Z_{a}^{i}=\tilde{Z}_{3}^{1 / 2}, & Z_{a}^{0}=Z_{11} \tilde{Z}_{3}^{1 / 2} / Z_{12} \\
Z_{\eta_{a}}=Z_{31}^{1 / 2}, & Z_{\psi}=Z_{\bar{\psi}}=\left(Z_{31} \tilde{Z}_{3} / Z_{2}\right)^{1 / 2} .
\end{array}
$$

From these relations and Eq. (34), we find $K_{B n} \chi_{B}^{n}=\left(Z_{31} \tilde{Z}_{3}\right)^{1 / 2} K_{n} \chi^{n}$, where summation over $n$ is not taken $(n=1,2, . ., 5)$. Note that the factor $\left(Z_{31} \tilde{Z}_{3}\right)^{1 / 2}$ is common for all $n$. This fact, together with the relation $\ell=\mathcal{L}_{\text {eff }}\left(A_{B}, \eta_{B}, \bar{\eta}_{B}, \psi_{B}, \bar{\psi}_{B} ; g_{B}, m_{B}, \alpha_{B}\right)$ obtained above, justifies [10] a posteriori the recursive procedure in $\S 3$.

It should be emphasized that we have uniquely deduced the results (38), which is in contrast with those in [2].

Derivation of $\tilde{Z}_{1}=Z_{12} / Z_{31}$

Following [2], we start with the Ward identity written in terms of the bare quantities:

$$
\int d^{3} x\left[\frac{\delta_{R} \tilde{\Gamma}_{B}}{\delta \chi_{B}^{n}} \frac{\delta_{L} \tilde{\Gamma}_{B}}{\delta K_{B n}}\right](x)=\partial_{0} \int d^{3} x\left[\eta_{B a} \frac{\delta_{R} \tilde{\Gamma}_{B}}{\delta A_{B a}^{0}}+K_{A_{B a}^{0}} \frac{\delta_{L} \tilde{\Gamma}_{B}}{\delta K_{\eta_{B a}}}\right](x) .
$$


According to our results on renormalization, the effective action $\tilde{\Gamma}_{B}$ is finite [2] when expressed in terms of renormalized quantities,

$$
\tilde{\Gamma}_{B}\left(\chi_{B}, \bar{\eta}_{B}, K_{B} ; g_{B}, m_{B}, \alpha_{B}\right)=\tilde{\Gamma}(\chi, \bar{\eta}, K ; g, m, \alpha) .
$$

Through this change of quantities, Eq. (39) turns out to

$$
\int d^{3} x\left[\frac{\delta_{R} \tilde{\Gamma}}{\delta \chi^{n}} \frac{\delta_{L} \tilde{\Gamma}}{\delta K_{n}}\right](x)=\frac{Z_{31} \tilde{Z}_{1}}{Z_{12}} \partial_{0} \int d^{3} x\left[\eta_{a} \frac{\delta_{R} \tilde{\Gamma}}{\delta A_{a}^{0}}+K_{A_{a}^{0}} \frac{\delta_{L} \tilde{\Gamma}}{\delta K_{\eta_{a}}}\right](x) .
$$

Since the left-hand side of this equation is UV finite, $Z_{31} \tilde{Z}_{1} / Z_{12}$ must be finite. This implies that in the recursive procedure described in $\S 3$, the UV-divergent part of $Z_{31} \tilde{Z}_{1}$ is equal to that of $Z_{12}$ in each loop order. Then, we obtain the relation

$$
\tilde{Z}_{1}=\frac{Z_{12}}{Z_{31}}=\frac{1}{D^{\prime}}
$$

where use has been made of Eq. (32).

\section{Strict Coulomb gauge}

In Appendices B and D, we show that, in the strict Coulomb gauge, $\tilde{Z}_{1}=D^{\prime}=1$, which is in accord with Eq. (41). Using this in Eq. (32), we obtain

$$
Z_{12}=Z_{42}=Z_{31}, \quad Z_{13}=Z_{32}, \quad Z_{\psi 12}=Z_{2}
$$

Then, the relation $g_{B} A_{B a}^{0}=g A_{a}^{0}$ (cf. after Eq. (34)) and then

$$
g_{B}^{2} D_{B}^{00}=g^{2} D^{00}
$$

hold, where $D^{00}$ is the time-time component of the gluon propagator.

It is worth mentioning here that, in the Hamiltonian, first-order, formalism [2], the identity (42) is deduced from the Ward identity. In contrast, as seen above, for deriving Eq. (42) in the Lagrangian formalism dealt with here, use of the Ward identity is not necessary. As a matter of fact, $D^{\prime}=1$ and $\tilde{Z}_{1}=1$ plays a role. The former is derived from the Zinn-Justin equation in Appendix B and the latter is obtained in Appendix D through formal diagrammatic analysis as in the case of Landau gauge. Physical importance of the relation (42) is fully discussed in $[1,2]$.

As a matter of course the Ward identity contains richer informations than the Zinn-Justin equation, so that different relations are to be obtained between various (renormalized) amplitudes. 
One-loop wave-function renormalization constants and $\delta m$ in the strict Coulomb gauge

As an illustration, we display here the results for the wave-function renormalization constants and $\delta m$ to one-loop order in the strict Coulomb gauge (see Appendix C):

$$
\begin{aligned}
Z_{31} & =-\frac{3 g^{2}}{8 \pi^{2}} \frac{1}{d-4}+\frac{g^{2}}{12 \pi^{2}} \frac{1}{d-4} \\
Z_{32} & =-\frac{7 g^{2}}{8 \pi^{2}} \frac{1}{d-4}+\frac{g^{2}}{12 \pi^{2}} \frac{1}{d-4} \\
Z_{33} & =-\frac{11 g^{2}}{8 \pi^{2}} \frac{1}{d-4}+\frac{g^{2}}{12 \pi^{2}} \frac{1}{d-4} \\
\tilde{Z}_{3} & =-\frac{g^{2}}{2 \pi^{2}} \frac{1}{d-4} \\
Z_{2} & =\frac{g^{2}}{6 \pi^{2}} \frac{1}{d-4} \\
\delta m & =-\frac{g^{2}}{2 \pi^{2}} \frac{1}{d-4} m .
\end{aligned}
$$

The second terms on the right-hand sides of Eqs. (43) - (45) come from the quark-loop diagram. These $Z$ 's and $\tilde{Z}_{3}$ satisfy the identities (32).

We explicitly confirmed in the Lagrangian formalism, as in other literatures in different formalisms, the absence of energy divergence in one-loop order (Appendix C).

\section{Summary and discussion}

In this paper we have addressed the problem of renormalizability of the Coulombgauge QCD within the Lagrangian, second-order, formalism. Starting with the ZinnJustin equation and following the procedure as in [11], we have proved a formal or algebraic renormalizability. The renormalization constants for the external sources $K_{a}^{i}, K_{a}^{0}, K_{\eta_{a}}, K_{\psi}$, and $K_{\bar{\psi}}$, which couple, respectively, to the composite operators $s A_{a}^{i}, s A_{a}^{0}, s \eta_{a}, s \psi$, and $s \bar{\psi}$ are uniquely determined.

We have derived the Ward identity (15) and from which we have obtained the

identity $\tilde{Z}_{3}=Z_{32} / Z_{31}$. With the aid of the Zinn-Justin equation, we have shown that $g A_{a}^{0}$ is unchanged under renormalization. In $[1,2]$, this is derived using the Ward 
identity. In contrast, in the present Lagrangian formalism, use of the Zinn-Justin equation is sufficient for proving this.

\section{Acknowledgments}

I wish to thank M. Inui and H. Kohyama for useful discussions. This work has been supported in part by the Grant-in-Aid for Scientific Research [(C)(2) No. 17540271] from the Ministry of Education, Culture, Sports, Science and Technology, Japan, No.(C)(2)-17540271.

\section{A Ward identity and Zinn-Justin equation}

\section{$\bar{\eta}_{a}$-dependence of $\Gamma$}

Changing the integration variable $\bar{\eta}_{a}^{\prime} \rightarrow \bar{\eta}_{a}^{\prime}+\delta \bar{\eta}_{a}^{\prime}$ in Eq. (12), we obtain

$$
\begin{aligned}
0 & =\int \mathcal{D}\left(\chi^{\prime}, \bar{\eta}^{\prime}\right) \delta \bar{\eta}_{a}^{\prime}\left[-\partial_{j} D_{a b}^{j}\left[A^{\prime}\right] \eta_{b}^{\prime}+\frac{\delta_{R} \Gamma}{\delta \bar{\eta}_{a}}\right] e^{i \int d^{4} y(\ldots)} \\
& =\int \mathcal{D}\left(\chi^{\prime}, \bar{\eta}^{\prime}\right) \delta \bar{\eta}_{a}^{\prime}\left[-\partial^{j} \frac{\delta_{L}}{i \delta K_{a}^{j}}+\frac{\delta_{R} \Gamma}{\delta \bar{\eta}_{a}}\right] e^{i \int d^{4} y(\ldots)}
\end{aligned}
$$

From this equation, we get

$$
\partial_{j} \frac{\delta_{L} \Gamma}{\delta K_{a}^{j}}+\frac{\delta_{L} \Gamma}{\delta \bar{\eta}_{a}}=0
$$

This relation tells us that $\Gamma$ depends on $\bar{\eta}$ only through $K_{a}^{j}+\partial^{j} \bar{\eta}_{a}$.

\section{Derivations of Ward identity and Zinn-Justin equation}

We introduce infinitesimal variations $[1,2]$

$$
\begin{aligned}
\chi^{\prime n}(x) & \rightarrow \chi^{\prime n}+f\left(x_{0}\right) s \chi^{\prime n}(x) \zeta \quad(n=1, \ldots, 5), \\
\bar{\eta}_{a}^{\prime}(x) & \rightarrow \bar{\eta}_{a}^{\prime}(x)+f\left(x_{0}\right) s \bar{\eta}_{a}^{\prime}(x) \zeta
\end{aligned}
$$

where $s \chi^{\prime n}$ and $s \bar{\eta}_{a}^{\prime}$ are as in Eqs. (8) - (11), and $f\left(x_{0}\right)$ is a $x_{0}$-dependent function. When $f$ is a constant, it reduces to the BRST transformation. Making this change 
of variables in Eq. (12), we obtain

$$
\begin{aligned}
& \int \mathcal{D}\left(\chi^{\prime}, \bar{\eta}^{\prime}\right) {\left[\int d^{4} x\left\{-\left(\frac{\delta_{R} \Gamma}{\delta \chi^{n}} s \chi^{\prime n}+\frac{\delta_{R} \Gamma}{\delta \bar{\eta}_{a}} s \bar{\eta}_{a}^{\prime}\right) f+\rho\left[\chi^{\prime}\right] \partial_{0} f\right\}\right] } \\
& \times \exp \left[i \int d^{4} y\left(\mathcal{L}_{\mathrm{eff}}\left(\chi^{\prime}, \bar{\eta}^{\prime}\right)+K_{n} s \chi^{\prime n}-\frac{\delta_{R} \Gamma}{\delta \chi^{n}}\left(\chi^{\prime n}-\chi^{n}\right)-\frac{\delta_{R} \Gamma}{\delta \bar{\eta}_{a}}\left(\bar{\eta}_{a}^{\prime}-\bar{\eta}_{a}\right)\right)\right] \\
&=0
\end{aligned}
$$

where $\rho$ is the BRST charge:

$$
\rho\left[\chi^{\prime}\right]=-F_{a}^{\prime 0 i} s A_{a i}^{\prime}+i \bar{\psi}^{\prime} \gamma_{0} s \psi^{\prime}+K_{a}^{0} s \eta_{a}^{\prime}
$$

We rewrite the term being proportional to $\rho \partial_{0} f$ as

$$
\begin{aligned}
\int \mathcal{D}\left(\chi^{\prime}, \bar{\eta}^{\prime}\right) & {\left[\int d^{4} x\left(-F_{a}^{\prime 0 i} s A_{a i}^{\prime}+i \bar{\psi}^{\prime} \gamma_{0} s \psi^{\prime}+K_{a}^{0} s \eta_{a}^{\prime}\right) \partial_{0} f\right] e^{i \int d^{4} y(\ldots)} } \\
= & -\int \mathcal{D}\left(\chi^{\prime}, \bar{\eta}^{\prime}\right) \exp \left[-i \int d^{4} y \frac{\delta_{R} \Gamma}{\delta A_{a}^{0}}\left(A_{a 0}^{\prime}-A_{a 0}\right)\right] \\
\times & {\left[\int d^{4} x\left(\eta_{a}^{\prime} \frac{\delta}{i \delta A_{a 0}^{\prime}}+K_{a}^{0} s \eta_{a}^{\prime}\right) \partial_{0} f\right] } \\
\times & \exp \left[i \int d ^ { 4 } y \left(\mathcal{L}_{\mathrm{eff}}\left(\chi^{\prime}, \bar{\eta}^{\prime}\right)+K_{n} s \chi^{\prime n}\right.\right. \\
& \left.\left.-\sum_{n \neq 2} \frac{\delta_{R} \Gamma}{\delta \chi^{n}}\left(\chi^{\prime n}-\chi^{n}\right)-\frac{\delta_{R} \Gamma}{\delta \bar{\eta}_{a}}\left(\bar{\eta}_{a}^{\prime}-\bar{\eta}_{a}\right)\right)\right]
\end{aligned}
$$

Carrying out the partial integration, we obtain

$$
\text { Eq. }(\mathrm{A} .4)=-\int \mathcal{D}\left(\chi^{\prime}, \bar{\eta}^{\prime}\right)\left[\int d^{4} x\left(\eta_{a}^{\prime} \frac{\delta_{R} \Gamma}{\delta A_{a}^{0}}+K_{a}^{0} \frac{\delta_{L} \Gamma}{\delta K_{\eta_{a}}}\right) \partial_{0} f\right] e^{i \int d^{4} y(\ldots)} .
$$

From the definition of $\eta_{a}$, we can make a replacement $\eta_{a}^{\prime} \rightarrow \eta_{a}$.

From here, we proceed as follows: i) Substitute Eq. (A.5) in Eq. (A.3), ii) translate to $\tilde{\Gamma}$ by Eq. (14), and iii) integrate by part with respect to $x_{0}$ for the term that involves $\partial_{0} f$. Then, using the arbitrariness of $f\left(x_{0}\right)$, we finally obtain the Ward identity (15),

$$
\int d^{3} x\left[\frac{\delta_{R} \tilde{\Gamma}}{\delta \chi^{n}} \frac{\delta_{L} \tilde{\Gamma}}{\delta K_{n}}\right](x)=\partial_{0} \int d^{3} x\left[\eta_{a} \frac{\delta_{R} \tilde{\Gamma}}{\delta A_{a}^{0}}+K_{a}^{0} \frac{\delta_{L} \tilde{\Gamma}}{\delta K_{\eta_{a}}}\right](x) .
$$

Integration of Eq. (A.6) over $x_{0}$ yields the Zinn-Justin equation (16). Eq. (A.6) holds for arbitrary $\alpha$ (gauge parameter). 


\section{B Derivation of $Z_{31} / Z_{12}=D^{\prime}=1$ in the strict Coulomb gauge}

By functionally differentiating the Zinn-Justin equation (16) with respect to $A_{a}^{\mu}, A_{b}^{\nu}$, and $\eta_{c}$, we obtain [13], after Fourier transformation,

$$
P_{\mu} \Pi^{\nu \mu}(P)=\Pi^{\nu \mu}(P) \tilde{\Pi}_{\mu}(P)
$$

which is diagonal in color space, so that the color index is dropped. $\Pi^{\nu \mu}$ is the twopoint gluon effective action, from which the gauge-fixing term is dropped. $\tilde{\Pi}_{\mu}(P)$ is defined by

$$
i g f_{a b c}\left\langle A_{b}^{\mu}(x) \eta_{c}(x) \bar{\eta}_{d}(y)\right\rangle_{t r} \stackrel{\text { F.T. }}{\longrightarrow} \delta_{a d} \tilde{\Pi}^{\mu}(P)
$$

where the suffix "tr" stands for truncation and "F.T." means to take Fourier transformation. The FP-ghost self-energy part $\tilde{\Pi}(P)$ is related to $\tilde{\Pi}_{\mu}(P)$ through $p^{i} \tilde{\Pi}_{i}(P)=$ $\tilde{\Pi}(P)$.

Let us introduce a tensor decomposition of $\Pi^{\nu \mu}$ :

$$
\Pi^{\mu \nu}(P)=g_{i}^{\nu} g_{j}^{\mu} g^{i j} A+p^{\underline{\nu}} p^{\underline{\mu}} B+p_{0}\left(n^{\nu} p^{\underline{\mu}}+p^{\underline{\nu}} n^{\mu}\right) C+n^{\nu} n^{\mu} E,
$$

where $n^{\mu}=(1,0)$ and $p^{\underline{\mu}}=p^{\mu}-p_{0} n^{\mu}$. Substitution of $\Pi_{B}^{\nu \mu}$, which is written in terms of bare quantities, into the "bare counterpart" of Eq. (B.1) yields two equations, one of which reads

$$
\left(p^{2}+\tilde{\Pi}_{B}\right) C_{B}-\left(1-\frac{\tilde{\Pi}_{B 0}}{p_{0}}\right) E_{B}=0 .
$$

Another equation leads to the same result as the one obtained below.

When expressed in terms of renormalized quantities, this equation should become [2] an UV-divergence free equation. Relations between the bare- and renormalizedquantities are $C_{B}=\left(Z_{31} Z_{33}\right)^{-1 / 2} C, E_{B}=Z_{33}^{-1} E$, and $p^{2}+\tilde{\Pi}_{B}=\tilde{Z}_{3}^{-1}\left(p^{2}+\tilde{\Pi}\right)$. In Appendix D, we show in the strict Coulomb gauge that $\tilde{\Pi}_{0}$ is UV finite and $\tilde{Z}_{1}=1$. Then,

$$
\tilde{\Pi}_{B 0}=\frac{1}{\tilde{Z}_{3}}\left(\tilde{Z}_{3} Z_{33}^{1 / 2} \frac{g_{B}}{g}\right) \tilde{\Pi}_{0}=\frac{1}{D^{\prime}} \tilde{\Pi}_{0},
$$

where $g_{B}$ is $g_{B}$ on the left-hand side of the "bare counterpart" of Eq. (B.2). Then, Eq (B.4) turns out to

$$
\left(p^{2}+\tilde{\Pi}\right) C=\tilde{Z}_{1} D^{\prime}\left(1-\frac{\tilde{\Pi}_{0}}{p_{0} D^{\prime}}\right) E
$$


Since the left-hand side is UV finite, same reasoning as in $\S 4$ (cf. Eq. (40)) leads to

$$
\tilde{Z}_{1}=D^{\prime}=1
$$

These relations holds for the strict Coulomb gauge and are in accord with Eq. (41).

\section{Derivation of Eqs. (43) - (45)}

The strict Coulomb gauge gluon propagator is given by Eq. (5) with $\alpha=0$. Straightforward computation using Eqs. (5) with $\alpha=0$, (7), and the forms of 3- and 4-gluon vertices yields, for the one-loop UV-divergent contributions to $\Pi^{\mu \nu}(Q)$ (Eq. (B.3)) (see, also, $[1,9]$ ),

$$
\begin{aligned}
A & =\frac{g^{2}}{120 \pi^{2}} \frac{1}{d-4}\left[\left(15 Q^{2}+96 q^{2}\right)-\left(60 Q^{2}+96 q^{2}\right)+10 Q^{2}\right] \\
B & =\frac{g^{2}}{120 \pi^{2}} \frac{1}{d-4}[93-48-10] \\
C & =\frac{g^{2}}{24 \pi^{2}} \frac{1}{d-4}[-3+24-2] \\
E & =\frac{g^{2}}{24 \pi^{2}} \frac{1}{d-4}[-15+48-2] q^{2} .
\end{aligned}
$$

Here, each term on the right-hand side of each equation is the contribution from the following one-loop diagrams:

- First term $\leftarrow$ The diagram that includes two transverse-gluon propagators.

- Second term $\leftarrow$ The diagram that includes one transverse-gluon propagator and one $A_{0}$ propagator and the tadpole diagram.

- Third term $\leftarrow$ The diagram with a quark loop.

From Eqs. (C.1) and (31), we extract Z's, as given in Eqs. (43) - (45) in the text. We briefly describe how Eq. (C.1) is derived. The (1)-type energy-diverging integrations appear. As mentioned in $\S 5$, it is known that, when all relevant contributions are added, cancellation occurs between them. Here, we substantiate this for the one-loop gluon two-point function in the Lagrangian formalism. Energy divergences 
arise only in the transverse-gluon two-point function. Explicit computation of the energy-divergent contributions of the type (1) yields

$$
\begin{aligned}
& \left(\Pi_{a b}^{i j}\right)_{1} \simeq 3 g^{2} \delta_{a b} \int \frac{d^{4} P}{(2 \pi)^{4}} \frac{1}{(\mathbf{k}-\mathbf{p})^{2}}\left(\delta^{i j}-\frac{p^{i} p^{j}}{p^{2}}\right) \\
& \left(\Pi_{a b}^{i j}\right)_{2}=-3 g^{2} \delta_{a b} \delta^{i j} \int \frac{d^{4} P}{(2 \pi)^{4}} \frac{1}{(\mathbf{k}-\mathbf{p})^{2}} \\
& \left(\Pi_{a b}^{i j}\right)_{3}=6 g^{2} \delta_{a b} \int \frac{d^{4} P}{(2 \pi)^{4}} \frac{p^{i} p^{j}}{p^{2}(\mathbf{k}-\mathbf{p})^{2}} \\
& \left(\Pi_{a b}^{i j}\right)_{4}=-3 g^{2} \delta_{a b} \int \frac{d^{4} P}{(2 \pi)^{4}} \frac{p^{i} p^{j}}{p^{2}(\mathbf{k}-\mathbf{p})^{2}}
\end{aligned}
$$

Here $\simeq$ indicates the energy-divergent contribution, and $\Pi_{j}(j=1, \ldots, 4)$ is the contribution from the following one-loop diagrams:

- $\Pi_{1} \leftarrow$ The diagram that includes one transverse-gluon propagator and one $A_{0}$ propagator.

- $\Pi_{2} \leftarrow$ The diagram that includes one $A_{0}$ propagator (tadpole diagram).

- $\Pi_{3} \leftarrow$ The diagram that includes two $A_{0}$ propagators.

- $\Pi_{4} \leftarrow$ The diagram with FP-ghost loop.

It can readily be seen that the cancellation occurs between the four contributions in Eq. (C.2).

The ill-defined integrals like (2) also appear. As mentioned in $§ 5$, such integrals can be set equal to zero.

\section{Diagrammatic analyses}

In this Appendix, we formally carry out some diagrammatic analyses. We start with following observations. Consider a diagram $G$ that includes FP-ghost external lines.

a) From Eq. (7), we see that the vertex factor for the external $\bar{\eta} A^{j} \eta$-vertex from which the outgoing ghost goes out is independent of the internal loop momenta. 
b) Strict Coulomb-gauge case: From Eq. (7), the vertex factor for the external $\bar{\eta}(P-$ Q) $A^{j}(Q) \eta(P)$-vertex, into which the incoming ghost enters, is proportional to $p_{j}-q_{j} . A^{j}(Q)$ constitutes the gluon propagator (5), the transverse part of which includes $\delta^{j i}-q^{j} q^{i} / q^{2}$. Then we have $\left(p_{j}-q_{j}\right)\left(\delta^{j i}-q^{j} q^{i} / q^{2}\right)=p_{j}\left(\delta^{j i}-q^{j} q^{i} / q^{2}\right)$, so that this vertex factor turns out to be independent of the internal loop momenta.

\section{UV divergent contribution to the ghost propagator $\tilde{\Pi}(P)$}

Consider a diagram $G$ that contributes to $\tilde{\Pi}(P)$. The vertex factor (Eq. (7)) for the external $\bar{\eta}(P) A^{i} \eta$-vertex from which the outgoing ghost goes out is proportional to $p^{i}$. The vertex factor for another external $\bar{\eta}(Q) A^{j} \eta$-vertex, into which the incoming ghost enters, is proportional to $q^{j}$. Because of the rotation symmetry, we have, after loop-integration, $\tilde{\Pi}(P) \propto p^{2} \mathcal{F}$, where $\mathcal{F}$ is a dimensionless function of $p_{0}, p$, and $m$. Since we have assumed that the divergent part of $\tilde{\Pi}$ is a local function of the fields (cf. after Eq. (18)), we have $\tilde{\Pi}^{(\infty)}(P) \propto p^{2} /(d-4)^{n}(n=1,2, \ldots)$, so that $\tilde{\mathcal{L}}^{(\epsilon)}$ in Eq. (21) does not involve the term $\left(\partial_{0} \bar{\eta}\right)\left(\partial_{0} \eta\right)$.

\section{UV finiteness of $\bar{\eta} A^{0} \eta$ three-point functions}

Let $\mathcal{F}(P, Q)$ be a $\bar{\eta} A^{0}(Q) \eta(P)$ three-point function. From its Lorentz structure, $\mathcal{F}$ is of the form $\mathcal{F}=\mathcal{F}_{1} p_{0}+\mathcal{F}_{2} q_{0}$. This, together with the above observation a), shows that the degree of UV divergence is -1 , so that $\mathcal{F}$ is UV finite. Then, $\tilde{\mathcal{L}}^{(\epsilon)}$ in Eq. (21) does not involve the term $\left(\partial_{0} \bar{\eta}\right) A^{0} \eta$.

\section{UV finiteness of $\tilde{\Pi}_{0}(P)$ in strict Coulomb gauge}

Consider a diagram $G$ that contributes to $\tilde{\Pi}_{0}(P)$, Eq. (B.2) with $\mu=0$. From the Lorentz structure $\tilde{\Pi}_{0}(P) \propto p_{0}$. This, together with the above observations a) and b), shows the degree of UV divergence of the diagram is -1 .

\section{$\tilde{Z}_{1}=1$ in strict Coulomb gauge}

Consider an $\bar{\eta} A^{j} \eta$ three-point function in the strict Coulomb gauge. Above observations a) and b) tell us that the $\bar{\eta} A^{j} \eta$ three-point function is UV finite and then, in the minimal subtraction scheme, $\tilde{Z}_{1}=1$, as in the Landau gauge case. 


\section{References}

[1] D. Zwanziger, Nucl. Phys. B518, 237 (1998).

[2] L. Baulieu and D. Zwanziger, Nucl. Phys. B548, 527 (1999).

[3] P. Doust, Ann. Phys. 177 (1987) 169.

[4] H. Cheng and E.-C. Tsai, Phys. Lett. 176 (1986) 130; Phys. Rev. D 40 (1989) 1246.

[5] H. Cheng and E.-C. Tsai, Phys. Rev. Lett. 57 (1986) 511.

[6] N. H. Christ and T. D. Lee, Phys. Rev. D 22 (1980) 939.

See, also, J. Schwinger, Phys. Rev. 127 (1962) 324.

[7] A. Andraši and J. C. Taylor, Eur. Phys. J. C41, 377 (2005).

[8] E. Braaten and R. D. Pisarski, Nucl. Phys. B337, 569 (1990).

[9] A, Andraši, Eur. Phys. J. C37, 307 (2004).

[10] C. Itzykson and J.-B. Zuber, Quantum Field Theory (McGraw-Hill Inc. 1980).

[11] S. Weinberg, The Quantum Theory of Fields (Vol. II) (Cambridge University Press 1996).

[12] R. Kobes, G. Kunstatter, and A. Rebhan, Nucl. Phys. B355, 1 (1991).

[13] M. Dirks, A. Niégawa and K. Okano, Phys. Lett. B461, 131 (1999). 